\title{
Synthesis of achiral and new chiral crown ethers containing a triphenylphosphane unit
}

\author{
Tamás Szabó, ${ }^{\mathrm{a}}$ László Petri, ${ }^{\mathrm{a}}$ Szilveszter Gergely, ${ }^{\mathrm{b}}$ and Péter Huszthy*a \\ ${ }^{a}$ Department of Organic Chemistry and Technology, Budapest University of Technology and \\ Economics, PO Box 91, H-1521 Budapest, Hungary \\ ${ }^{b}$ Department of Applied Biotechnology and Food Science, Budapest University of Technology \\ and Economics, PO Box 91, H-1521 Budapest, Hungary \\ E-mail: huszthy@mail.bme.hu
}

DOI: $\underline{\text { http://dx.doi.org/10.3998/ark.5550190.p009.075 }}$

\begin{abstract}
Synthesis and characterization of achiral $\mathbf{1}$ and new chiral macrocycles $(R, R)-\mathbf{2},(S, S)-\mathbf{3}$ and $(S, S)$-4 containing a triphenylphosphanone unit have been accomplished. A new method was used for the reduction of phosphanones to phosphanes. Using this method new potential catalyst ligands 15, $(R, R)-\mathbf{1 6},(S, S)-\mathbf{1 7}$ and $(S, S)-\mathbf{1 8}$ containing a triphenylphosphane unit were synthesized and characterized. These ligands are promising candidates for coordination chemistry studies and homogeneous catalytic reactions.
\end{abstract}

Keywords: Macrocycles, triarylphosphanes, triarylphosphanones, crown ethers

\section{Introduction}

Trivalent phosphane compounds have gained great attention in organic chemistry since $1948^{1-8}$ due to their complexing ability and their potential use as catalysts in homogenous reactions, such as hydrogenation ${ }^{9,10}$ for example. Although P-heterocyclic ligands are becoming the subjects of a more and more important research area, ${ }^{11}$ there are only a few $\mathrm{P}(\mathrm{III})$-containing macrocycles reported in the literature due to the difficulties of their preparation. ${ }^{12-14}$ The advantageous features of these macrocycles are as follows. Beside the trivalent phosphorus, the oxygen atoms of the crown ethers can improve the complexation with transition metals. The macrocycle of the crown ethers containing the triphenylphosphane unit induces a rigid conformation, which can increase the selectivity of the catalysis.

A few years ago we reported the synthesis of crown ethers containing an ethyl diarylphosphinate moiety. ${ }^{15}$ In the present paper we describe a method for an ethyl diarylphosphinate-triarylphosphanone transformation including crown ether derivatives as well. 
Silane compounds are commonly used for the reduction of phosphanones to phosphanes. ${ }^{16-23}$ However, with most of these reported methods the phosphane is difficult to purify. There are reported reductions using triethoxysilane, but in all cases $\mathrm{Ti}$ compounds and a solvent, for example benzene are used. ${ }^{24-30}$ In this paper we report an easy way to deoxygenate phosphanones using trimethoxy- or triethoxysilanes without a solvent and a catalyst, gaining macrocycles $\mathbf{1}$, $(R, R)-\mathbf{2},(S, S)-\mathbf{3},(S, S)-\mathbf{4}$ containing a triphenylphosphane unit. Crown ethers $\mathbf{1}$ and $\mathbf{5}$ are already known compounds ${ }^{14}$, here we describe a new route for their preparation, which gives better yields. Enantiopure macrocycles $(R, R)-\mathbf{2},(S, S)-\mathbf{3},(S, S)-\mathbf{4},(R, R)-\mathbf{6},(S, S)-\mathbf{7}$, and $(S, S)-\mathbf{8}$ are new compounds.

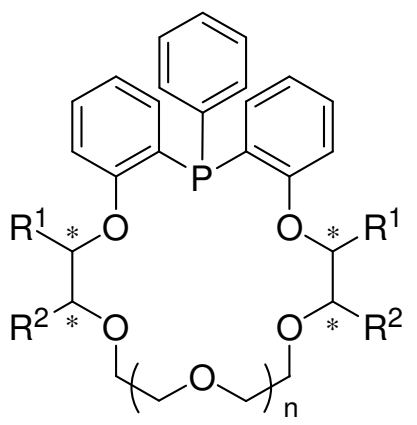

1: $R^{1}=R^{2}=H, n=1$

$(R, R)-2: \mathrm{R}^{1}=\mathrm{Me}, \mathrm{R}^{2}=\mathrm{H}, \mathrm{n}=1$

$(S, S)-3: R^{1}=H, R^{2}=$ Oct, $n=2$

$(S, S)-4: R^{1}=H, R^{2}=M e, n=1$

Figure 1. Structures of crown ethers 1- $(S, S)-\mathbf{4}$ containing a triphenylphosphane unit.

\section{Results and Discussion}

The preparation of ligands containing a phenyl diarylphosphanone unit $[\mathbf{5},(R, R)-\mathbf{6},(S, S)-\mathbf{7}$, and $(S, S)-8$ ] was achieved in two ways. Phosphanones 5 and $(R, R)-\mathbf{6}$ were respectively prepared from the already reported macrocycles $9^{15}$ and $(R, R)-\mathbf{1 0}^{15}$ via the appropriate phosphine chlorides with phenylmagnesium bromide adopting a known procedure ${ }^{31}$ (see Scheme 1). 
<smiles>[R]C(COCCOCCOCC([R])Oc1ccccc1P(=O)(OCC)OCC)Oc1ccccc1</smiles>

9: $\mathrm{R}=\mathrm{H}$

$(R, R)-10: \mathrm{R}=\mathrm{Me}$
1. $\mathrm{PCl}_{5}, \mathrm{CH}_{2} \mathrm{Cl}_{2}$ heat

2. $\mathrm{PhMgBr}$, ether toluene, $0^{\circ} \mathrm{C}$<smiles>[R]C(=O)COCCCOCCOCC([R])Oc1ccccc1P(=O)(c1ccccc1)c1ccccc1OC([R])CP</smiles>

5: $\mathrm{R}=\mathrm{H}(45 \%)$

$(R, R)-6: \mathrm{R}=\mathrm{Me}(89 \%)$

Scheme 1. Preparation of crown ethers containing a triphenylphosphanone unit.

It was planned to prepare new crown ethers $(S, S)-\mathbf{7}$ and $(S, S)-\mathbf{8}$ by macrocyclization of the reported phenyl bis(2-hydroxyphenyl)phosphanone $(\mathbf{1 1})^{32}$ and the reported enantiopure dimethyland dioctyl-substituted tetra- and pentaethylene glycol ditosylates ${ }^{33}[(S, S)-\mathbf{1 2},(S, S)-\mathbf{1 3}]$ at $80{ }^{\circ} \mathrm{C}$ in DMF using $\mathrm{K}_{2} \mathrm{CO}_{3}$ as a base (see Scheme 2).

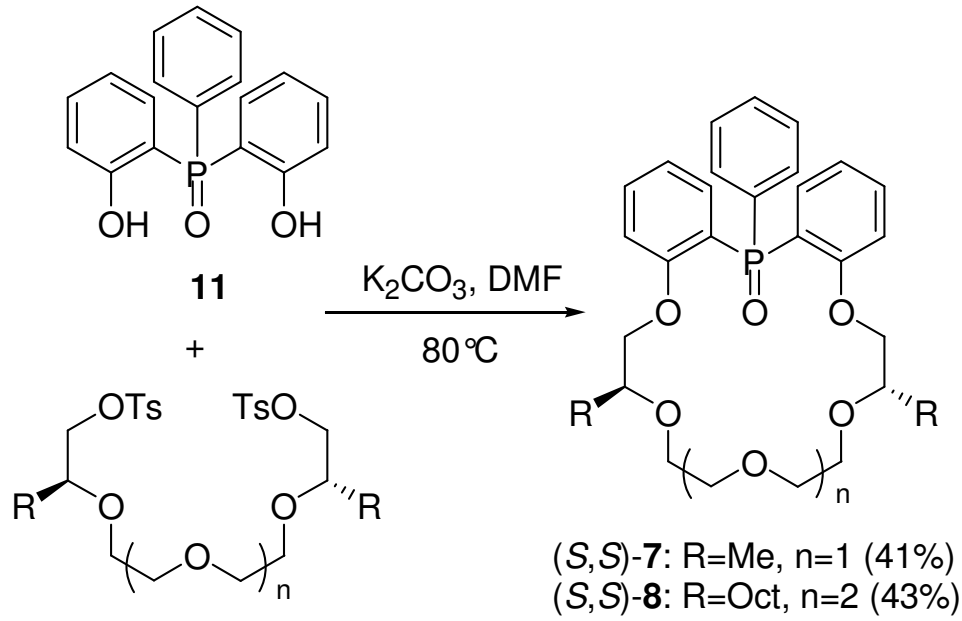

(S,S)-12: $\mathrm{R}=\mathrm{Me}, \mathrm{n}=1$

$(S, S)$-13: $R=$ Oct, $n=2$

Scheme 2. Preparation of crown ethers containing a triphenylphosphanone unit.

Although there is a reported procedure ${ }^{32}$ for the preparation of phosphanone 11, we could not reproduce it with acceptable yields, so we tried to find another way.

The free hydroxyl groups of the known ethyl bis(2-hydroxyphenyl)phosphinate (14) ${ }^{34}$ were protected by $O$-benzylation using benzyl bromide and $\mathrm{K}_{2} \mathrm{CO}_{3}$. $O$-benzyl ethyl phosphinate 15 was obtained in $71 \%$ yield and transformed to triphenylphosphanone derivative $\mathbf{1 6}$ using the same method as described above for $\mathbf{5}$ and $(R, R)-\mathbf{6}$. The benzyl protecting groups were removed by hydrogenolys in a very good yield (see Scheme 3 ). 
<smiles>CCOP(=O)(c1ccccc1O)c1ccccc1O</smiles>

14

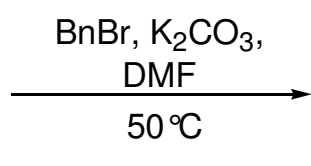

$50^{\circ} \mathrm{C}$<smiles>CCOc1ccccc1P(=O)(OCc1ccccc1)c1ccccc1OCc1ccccc1</smiles>

15 (71\%)

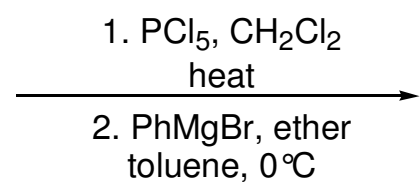

toluene, $0^{\circ} \mathrm{C}$

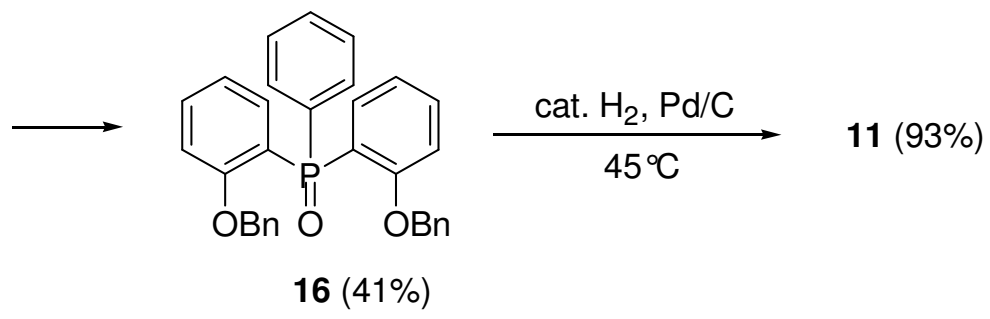

Scheme 3. Preparation of 11 bis(2-hydroxyphenyl)phenyl- $\lambda^{5}$-phosphanone.

There are numerous silane reducing agents for the preparation of phosphanes from phosphanones in the literature, but in most of those cases it is difficult to get the product from the reaction mixture, or to avoid using a solvent, such as benzene. ${ }^{24,28,29} \mathrm{We}$ tried to find an easy and simple way for the deoxygenation of phosphanones. First triphenylphosphanone (17) was used as a model compound to investigate the reduction. Triethoxysilane and trimethoxysilane were applied as the reducing agents in a sealed tube. The experiments showed that both reducing agents were effective, and the purification was easy in both cases. The silanes were both the reagent and the solvent, and after completion of the reaction the unreacted silanes were removed by simple evaporation under reduced pressure. Using trimethoxysilane we obtained a slightly better yield for $\mathbf{1 8}$ than applying triethoxysilane (see Scheme 4).<smiles>O=P(c1ccccc1)(c1ccccc1)c1ccccc1</smiles>

17

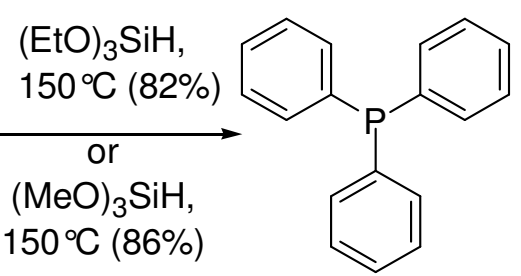

18

Scheme 4. Reduction of triphenylphosphanone to triphenylphosphane.

The reduction of the crown ethers containing the triarylphosphanone unit was carried out as well. Macrocycles 5, $(R, R)-\mathbf{6}$ and $(S, S)$-8 were deoxygenated using trimethoxysilane in good yields (see Scheme 5). 


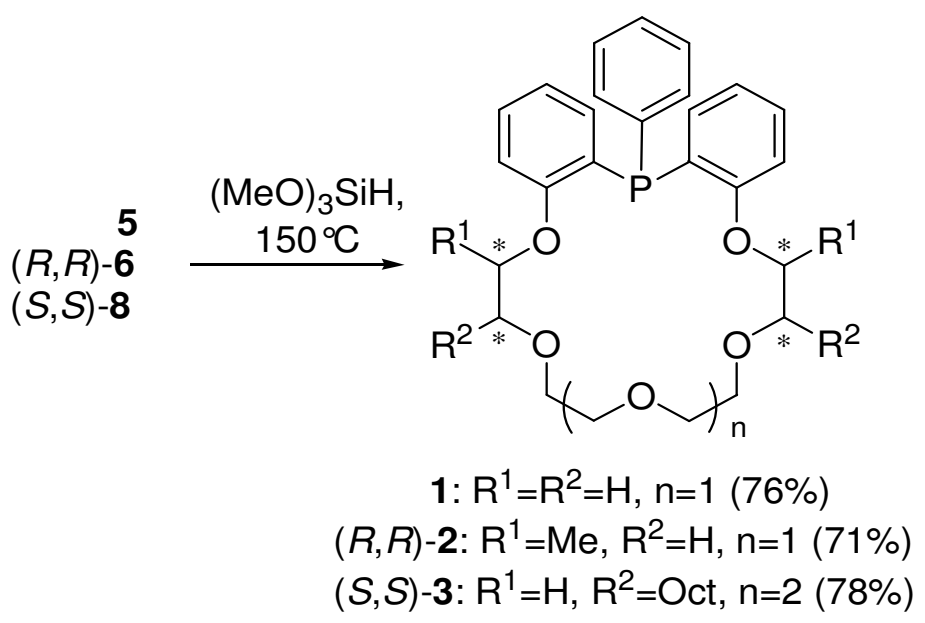

Scheme 5. Reduction of phosphanone-crown ethers using trimethoxysilane.

We wanted to know if the reduction procedure using triethoxysilane also works in the case of crown ethers. Macrocycle $(S, S)-7$ was also deoxygenated using triethoxysilane (see Scheme 6). Thus we can say that both reducing agents are suitable for the reduction of phosphanone-crown ethers as well.

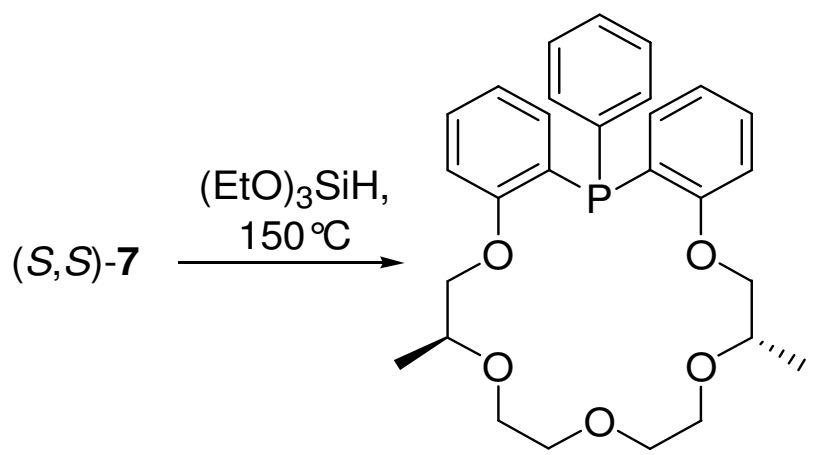

$(S, S)-4(56 \%)$

Scheme 6. Reduction of phosphanone crown ether $(S, S)-7$ using triethoxysilane.

Although macrocycles $\mathbf{1}$ and $\mathbf{5}$ were prepared earlier, the reported yields were rather low and the compounds were not well characterized. ${ }^{14}$

In our hands, the compounds are fully characterized. We note here that in the MS spectra of the phosphanes 1, $(R, R)-\mathbf{2},(S, S)-\mathbf{3}$ and $(S, S)-\mathbf{4}$ only the molecular peaks for the appropriate phosphanones could be found. The completion of the phosphanone-phosphane transformation was proved by ${ }^{31} \mathrm{P}-\mathrm{NMR}$ (the difference of the concerning $\delta$ values was larger than 45 ), IR (the lack of the peaks for phosphanones in the spectra of phosphanes) and TLC analysis (big differences in $R_{\mathrm{f}}$ values). 


\section{Conclusions}

The synthesis and characterization of achiral 1 and new enantiopure $(R, R)-\mathbf{2},(S, S)-\mathbf{3}$ and $(S, S)-\mathbf{4}$ crown ethers containing a triarylphosphane unit have been carried out successfully. The appropriate phosphanones were reduced to phosphanes applying trialkoxy silanes. As a reducing agent neat trimethoxysilane or triethoxysilane without any solvents and/or catalysts was used successfully. These macrocycles containing a triphenylphosphane unit are promising precursors of catalysts for transition metal catalytic reactions. We plan to study the metal ion complexing ability and homogeneous catalytic behavior of these crown ethers.

\section{Experimental Section}

General. Infrared spectra were recorded on a PerkinElmer Spectrum 400 FT-IR spectrometer, with Universal ATR Sampling Accessory, Composite Diamond/ZnSe Crystal. PerkinElmer Spectrum 6.3.2 software was used for data acquisition and qualitative analyses. Optical rotations were taken on a Perkin-Elmer 241 polarimeter that was calibrated by measuring the optical rotations of both enantiomers of menthol. ${ }^{1} \mathrm{H}-\mathrm{NMR}$ spectra were taken either on a Bruker DRX500 Avance spectrometer (500 MHz, reference: TMS) or on a Brucker 300 Avance spectrometer (300 MHz, reference: TMS) and it is indicated in each individual case. ${ }^{13} \mathrm{C}$-NMR spectra were taken either on a Bruker DRX-500 Avance spectrometer (125.8 MHz, reference: TMS) or on a Brucker 300 Avance spectrometer (75.5 MHz, reference: TMS) and it is indicated in each individual case. ${ }^{31} \mathrm{P}-\mathrm{NMR}$ spectra were recorded on a Brucker 300 Avance spectrometer (121.5 $\mathrm{MHz}$, reference: $\mathrm{H}_{3} \mathrm{PO}_{4}$ ). HPLC-DAD-MS/MS experiments were performed on an Agilent 1200 HPLC system(G1379B degasser, G1312B binary gradient pump, G1367C autosampler, G1316B column thermostat and G1315C diode array detector) coupled with an Agilent 6410 triple quadrupole mass spectrometer equipped with an ESI ion source (Agilent Technologies, Waldbronn, Germany). Masshunter B.03.01 software was used for data acquisition and qualitative analyses. Chiral HPLC measurements were carried out on a Phenomenex, Lux Cellulose-2 column $(5 \mu \mathrm{m}, 250 \times 6 \mathrm{~mm})$. Elemental analyses were performed in the Microanalytical Laboratory of the Department of Organic Chemistry, Institute of Chemistry, L. Eötvös University, Budapest, Hungary. Starting materials were purchased from Sigma-Aldrich Corporation unless otherwise noted. Melting points were taken on a Boetius micro-melting point apparatus and were uncorrected. Silica gel $60 \mathrm{~F}_{254}$ (Merck) plates were used for TLC. Silica gel 60 (70-230 mesh, Merck) were used for column chromatography. Silica gel $60 \mathrm{~F}_{254}$ and aluminium oxide $150 \mathrm{~F}_{254}$ (Merck) plates were used for PLC (preparative layer chromatography). Ratios of solvents for the eluents are given in volumes $(\mathrm{mL} / \mathrm{mL})$. Solvents were dried and purified according to well-established ${ }^{35}$ methods. Evaporations were carried out under reduced pressure unless otherwise stated. 
Ethyl bis(2-benzyloxyphenyl)phosphinate (15). Ethyl bis(2-hydroxyphenyl)phosphinate (14) ${ }^{34}$ $(10.00 \mathrm{~g}, 37 \mathrm{mmol})$, benzyl bromide $(10.77 \mathrm{~mL}, 90 \mathrm{mmol})$ and finely powdered anhydrous $\mathrm{K}_{2} \mathrm{CO}_{3}(49.67 \mathrm{~g}, 360 \mathrm{mmol})$ were mixed with vigorous stirring in dry DMF (330 mL) under Ar. The temperature of the reaction mixture was raised to $50{ }^{\circ} \mathrm{C}$ and kept stirring at this temperature until TLC analysis showed the total consumption of the starting materials (2 days). The solvent was removed at $40{ }^{\circ} \mathrm{C}$ and $\mathrm{CH}_{2} \mathrm{Cl}_{2}(400 \mathrm{~mL})$ and water $(300 \mathrm{~mL})$ were added to the residue. The phases were shaken well and separated. The aqueous phase was extracted with $\mathrm{CH}_{2} \mathrm{Cl}_{2}(3 \times 150$ $\mathrm{mL}$ ). The combined organic phase was dried over $\mathrm{MgSO}_{4}$, filtered and the solvent was removed. The crude product was recrystallized from toluene to give $15(11.63 \mathrm{~g}, 71 \%) \mathrm{mp} 111-113{ }^{\circ} \mathrm{C}$ (from toluene). $R_{\mathrm{f}}$ : 0.36 (silica gel TLC, acetone-hexane 1:2). ${ }^{1} \mathrm{H}-\mathrm{NMR}\left(500 \mathrm{MHz}, \mathrm{CDCl}_{3}\right) \delta$ $1.33\left(\mathrm{t}, J 7 \mathrm{~Hz}, 3 \mathrm{H}, \mathrm{CH}_{3}\right), 4.11\left(\mathrm{~m}, 2 \mathrm{H}, \mathrm{OCH}_{2}\right), 4.94\left(\mathrm{~s}, 4 \mathrm{H}, \mathrm{Ar} \mathrm{CH}_{2}\right), 6.85-6.91(\mathrm{~m}, 4 \mathrm{H}, \mathrm{ArH})$, 7.05-7.07 (m, 4H, ArH), 7.23-7.29 (m, 6H, ArH), 7.39-7.41 (m, 2H, ArH), 7.93-7.95 (m, 2H, $\mathrm{ArH}) .{ }^{13} \mathrm{C}-\mathrm{NMR}\left(75 \mathrm{MHz}, \mathrm{CDCl}_{3}\right) \delta 16.65\left(\mathrm{~d}, J 6.75 \mathrm{~Hz}, \mathrm{CH}_{3}\right), 60.55\left(\mathrm{~d}, J 6 \mathrm{~Hz}, \mathrm{OCH}_{2}\right), 70.09$ $\left(\mathrm{Ar} \mathrm{CH}_{2}\right), 112.01$ (d, J 8.25 Hz, ArC), 120.61 (d, $\left.J 12 \mathrm{~Hz}, \operatorname{ArC}\right), 120.81$ (d, $\left.J 140.25 \mathrm{~Hz}, \operatorname{ArC}\right)$, 127.41, 127.79, 128.40 (ArC), 133.54 (d, J $2.25 \mathrm{~Hz}, \mathrm{ArC}$ ), 135.42 (d, J $6 \mathrm{~Hz}, \mathrm{ArC}$ ), 136.48 (ArC), 160.05 (d, J 4.5 Hz, ArC). ${ }^{31} \mathrm{P}-\mathrm{NMR}\left(121.5 \mathrm{MHz}, \mathrm{CDCl}_{3}\right): \delta 26.23$; MS: $459.2(\mathrm{M}+\mathrm{H})^{+}$.

Bis(2-benzyloxyphenyl)phenyl- $\lambda^{\mathbf{5}}$-phosphanone (16). To the solution of phosphinate 15 (10.00 $\mathrm{g}, 21.8 \mathrm{mmol})$ in dry $\mathrm{CH}_{2} \mathrm{Cl}_{2}(330 \mathrm{~mL}) \mathrm{PCl}_{5}(4.86 \mathrm{~g}, 23.3 \mathrm{mmol})$ was added under Ar. The mixture was stirred at reflux temperature for 3 hours and then at $\mathrm{rt}$ for 15 minutes. The solvent was removed and dry toluene $(300 \mathrm{~mL})$ and dry ether $(100 \mathrm{~mL})$ were added to the residue under Ar. The stirred mixture was cooled to $0{ }^{\circ} \mathrm{C}$ and $\mathrm{PhMgBr}(43.4 \mathrm{mmol}, 29 \mathrm{~mL}, 1.5 \mathrm{M}$ solution in ether) was added dropwise. After stirring the reaction mixture for 10 minutes at $0{ }^{\circ} \mathrm{C}$ the temperature was raised to $\mathrm{rt}$ and it was kept stirring for 2 hours. The mixture was poured into icewater $(500 \mathrm{~mL})$ and $\mathrm{CH}_{2} \mathrm{Cl}_{2}(150 \mathrm{~mL})$ was added. The phases were shaken well, separated, and the organic phase was shaken with water $(2 \times 150 \mathrm{~mL})$, dried over $\mathrm{MgSO}_{4}$, filtered and the solvent was removed. The crude product was purified by chromatography on silica gel using acetonehexane mixtures (gradient elution with increasing polarity from 1:3 to 2:1) as eluents to give 16 $(4.41 \mathrm{~g}, 41 \%)$ as a white solid. $\mathrm{mp} 192-194{ }^{\circ} \mathrm{C}$ (from acetone); $R_{\mathrm{f}}: 0.29$ (silica gel TLC, acetonehexane 1:2). ${ }^{1} \mathrm{H}-\mathrm{NMR}\left(500 \mathrm{MHz}, \mathrm{CDCl}_{3}\right) \delta 4.90$ (s, 4H, $\mathrm{Ar} \mathrm{CH}_{2}$ ), 6.88-6.93 (m, 6H, ArH), 6.99$7.01(\mathrm{~m}, 2 \mathrm{H}, \mathrm{ArH}), 7.15-7.26$ (m, 8H, ArH), 7.38-7.47 (m, 3H, ArH), 7.66-7.77 (m, 4H, ArH); ${ }^{13} \mathrm{C}-\mathrm{NMR}\left(75 \mathrm{MHz}, \mathrm{CDCl}_{3}\right) \delta 70.34\left(\mathrm{Ar} \mathrm{CH}_{2}\right), 112.22(\mathrm{~d}, J 6 \mathrm{~Hz}, \mathrm{ArC}), 121.12(\mathrm{~d}, J 12 \mathrm{~Hz}$, ArC), 127.42, 127.83, 127.96, 128.43, 131.14 (ArC), 132.21 (d, J 10.5 Hz, ArC), 133.75 (ArC), 134.86 (d, $J 8.25 \mathrm{~Hz}, \mathrm{ArC}), 136.20,160.24$ (ArC). ${ }^{31} \mathrm{P}-\mathrm{NMR}\left(121.5 \mathrm{MHz}, \mathrm{CDCl}_{3}\right.$ ): $\delta$ 25.35; MS: $491.2(\mathrm{M}+\mathrm{H})^{+}$.

Bis(2-hydroxyphenyl)phenyl- $\lambda^{\mathbf{5}}$-phosphanone (11). To $\mathrm{Pd} / \mathrm{C}$ catalyst $(10 \% \mathrm{Pd}$ on charcoal, $1.20 \mathrm{~g}$ ) methanol $(10 \mathrm{~mL})$ was added under Ar. The Ar protecting gas was exchanged by $\mathrm{H}_{2}$ and the mixture was stirred under atmospheric $\mathrm{H}_{2}$ for 10 minutes. The solution of 16 (4.01 g, 8.20 $\mathrm{mmol})$ in methanol $(150 \mathrm{~mL})$ was added to the prehydrogenated catalyst and this mixture was stirred vigorously at $45^{\circ} \mathrm{C}$ until the consumption of $\mathrm{H}_{2}$ stopped. $\mathrm{H}_{2}$ was exchanged by $\mathrm{Ar}$ and the mixture was filtered using a celite pad. The solvent was removed to obtain pure 11 (2.36 g, 
93\%). mp 236-237 ${ }^{\circ} \mathrm{C}$ (from methanol). MS: $311.1(\mathrm{M}+\mathrm{H})^{+}$. Other physical and spectroscopic data were very similar to those of reported in the literature ${ }^{32}$.

\section{2-Phenyl-6,7,9,10,12,13,15,16-octahydro-22H-22 $\lambda^{5}$-dibenzo[n,q][1,4,7,10,13,16]pentaoxa-} phosphacyclooctadecin-22-one (5). To the solution of the reported macrocycle $\mathbf{9}^{15}(3.10 \mathrm{~g}, 7.1$ $\mathrm{mmol})$ in dry $\mathrm{CH}_{2} \mathrm{Cl}_{2}(100 \mathrm{~mL}) \mathrm{PCl}_{5}(1.63 \mathrm{~g}$, $7.8 \mathrm{mmol})$ was added under Ar. The mixture was stirred at reflux temperature for 3 hours and then at $\mathrm{rt}$ for 15 minutes. The solvent was removed and dry toluene $(100 \mathrm{~mL})$ and dry ether $(30 \mathrm{~mL})$ were added to the residue under Ar. The stirred mixture was cooled to $0{ }^{\circ} \mathrm{C}$ and $\mathrm{PhMgBr}(15.0 \mathrm{mmol}, 10 \mathrm{~mL}, 1.5 \mathrm{M}$ solution in ether) was added dropwise. After stirring the reaction mixture for 10 minutes at $0{ }^{\circ} \mathrm{C}$ the temperature of it was raised to $\mathrm{rt}$ and stirring was continued for 24 hours. The mixture was poured into ice-water (150 $\mathrm{mL})$ and $\mathrm{CH}_{2} \mathrm{Cl}_{2}(150 \mathrm{~mL})$ was added to it. The phases were shaken well and separated. The aqueous phase was shaken with $\mathrm{CH}_{2} \mathrm{Cl}_{2}(3 \times 50 \mathrm{~mL})$. The combined organic phase was dried over $\mathrm{MgSO}_{4}$, filtered and the solvent was removed. The crude product was purified by chromatography on silica gel using methanol- $\mathrm{CH}_{2} \mathrm{Cl}_{2}$ (1:30) as an eluent to give $\mathbf{5}(1.50 \mathrm{~g}, 45 \%)$ as a white solid. mp $173-175{ }^{\circ} \mathrm{C}$ (from methanol); $R_{\mathrm{f}}$ : 0.38 (silica gel TLC, methanol- $\mathrm{CH}_{2} \mathrm{Cl}_{2}$ $1: 20)$. IR (neat) $v_{\max } 1166 \mathrm{~cm}^{-1} v \mathrm{P}=\mathrm{O} ;{ }^{1} \mathrm{H}-\mathrm{NMR}\left(500 \mathrm{MHz}, \mathrm{CDCl}_{3}\right) \delta 3.22-3.54(\mathrm{~m}, 12 \mathrm{H}$, $\left.\mathrm{OCH}_{2}\right), 4.00-4.02\left(\mathrm{~m}, 2 \mathrm{H}, \mathrm{OCH}_{2}\right), 4.20-4.24\left(\mathrm{~m}, 2 \mathrm{H}, \mathrm{OCH}_{2}\right), 7.00-7.05(\mathrm{~m}, 4 \mathrm{H}, \mathrm{ArH}), 7.43-7.48$ $(\mathrm{m}, 5 \mathrm{H}, \mathrm{ArH}), 7.69-7.73(\mathrm{~m}, 2 \mathrm{H}, \mathrm{ArH}), 8.04-8.08$ (m, 2H, ArH). ${ }^{13} \mathrm{C}-\mathrm{NMR}\left(75 \mathrm{MHz}, \mathrm{CDCl}_{3}\right) \delta$ 66.52, 69.92, 70.81, $71.34\left(\mathrm{OCH}_{2}\right) .111 .86(\mathrm{~d}, J 6.6 \mathrm{~Hz}, \mathrm{ArC}), 120.39$ (d, J $\left.12.2 \mathrm{~Hz}, \operatorname{ArC}\right)$, 121.30 (d, $J 107.5 \mathrm{~Hz}, \operatorname{ArC}), 127.67$ (d, $J 12.9 \mathrm{~Hz}, \operatorname{ArC}), 130.68,130.71$ (ArC), 132.21 (d, $J 10.6$ $\mathrm{Hz}, \mathrm{ArC}$ ). 133.09, 133.11, 134.25, 134.35 (ArC), 160.27 (d, J 2.9 Hz, ArC). ${ }^{31} \mathrm{P}-\mathrm{NMR}$ (121.5 $\left.\mathrm{MHz}, \mathrm{CDCl}_{3}\right): \delta$ 24.90. MS: $469.2(\mathrm{M}+\mathrm{H})^{+}$. Anal Calcd for $\mathrm{C}_{26} \mathrm{H}_{29} \mathrm{O}_{6} \mathrm{P}: \mathrm{C}, 66.66 ; \mathrm{H}, 6.24$. Found: C, 66.55; H, 6.14.

$(6 R, 16 R)-6,16-D i m e t h y l-22-p h e n y l-6,7,9,10,12,13,15,16-o c t a h y d r o-22 H-22 \lambda^{5}$-dibenzo[n,q]$[1,4,7,10,13,16]$ pentaoxaphosphacyclooctadecin-22-one $[(\boldsymbol{R}, \boldsymbol{R})-6]$. Macrocycle $(R, R)-6$ was prepared from the reported crown ether $(R, R)-\mathbf{1 0}^{15}(0.252 \mathrm{~g}, 0.54 \mathrm{mmol})$ in the same way as described above for 5. The crude product was purified by PLC on silica gel using methanol$\mathrm{CH}_{2} \mathrm{Cl}_{2}$ (1:30) as an eluent to give $(R, R)-6(0.239 \mathrm{~g}, 89 \%)$ as a white solid. mp $168-170{ }^{\circ} \mathrm{C}$ (from methanol). $R_{\mathrm{f}}: 0.36$ (silica gel TLC, methanol- $\left.\mathrm{CH}_{2} \mathrm{Cl}_{2} 1: 20\right) ;[\alpha]_{\mathrm{D}}{ }^{34}-92.28$ (c 2.57, $\mathrm{CH}_{2} \mathrm{Cl}_{2}$ ); IR (neat) $v_{\max } 1163,1181 \mathrm{~cm}^{-1} v \mathrm{P}=\mathrm{O} .{ }^{1} \mathrm{H}-\mathrm{NMR}\left(300 \mathrm{MHz}, \mathrm{CDCl}_{3}\right) \delta 1.01\left(\mathrm{~d}, J 6.2 \mathrm{~Hz}, 3 \mathrm{H}, \mathrm{CH}_{3}\right)$, $1.13\left(\mathrm{~d}, J 6.3 \mathrm{~Hz}, 3 \mathrm{H}, \mathrm{CH}_{3}\right), 2.57-2.66\left(\mathrm{~m}, 1 \mathrm{H}, \mathrm{OCH}, \mathrm{OCH}_{2}\right), 3.01-3.03\left(\mathrm{~m}, 2 \mathrm{H}, \mathrm{OCH}, \mathrm{OCH}_{2}\right)$, 3.10-3.15 (m, 1H, OCH, $\left.\mathrm{OCH}_{2}\right), 3.23-3.30\left(\mathrm{~m}, 2 \mathrm{H}, \mathrm{OCH}, \mathrm{OCH}_{2}\right), 3.39-3.62(\mathrm{~m}, 6 \mathrm{H}, \mathrm{OCH}$, $\left.\mathrm{OCH}_{2}\right), 4.47-4.60$ (m, 2H, OCH, $\left.\mathrm{OCH}_{2}\right), 6.83-6.91$ (m, 1H, ArH), 6.97-7.05 (m, 3H, ArH), 7.18$7.25(\mathrm{~m}, 1 \mathrm{H}, \mathrm{ArH}), 7.35-7.48(\mathrm{~m}, 5 \mathrm{H}, \mathrm{ArH}), 7.98-8.04(\mathrm{~m}, 3 \mathrm{H}, \mathrm{ArH}) .{ }^{13} \mathrm{C}-\mathrm{NMR}(75 \mathrm{MHz}$, $\left.\mathrm{CDCl}_{3}\right) \delta 16.76,17.74\left(\mathrm{CH}_{3}\right), 70.68,70.95,71.21,71.39,71.67,73.68,73.77,74.54(\mathrm{OCH}$, $\mathrm{OCH}_{2}$ ), 105.07 (ArC), 111.71 (d, J 6.8 Hz, ArC), 113.14 (d, J 6.3 Hz, ArC), 119.99 (d, J 12.8 $\mathrm{Hz}, \operatorname{ArC}), 120.35$ (d, J 11.9 Hz, ArC), 122.48 (d, J 108.3 Hz, ArC), 124.06 (d, J 108.9 Hz, ArC), 127.98 (d, J $12.4 \mathrm{~Hz}, \operatorname{ArC}), 131.28$ (d, J $2.7 \mathrm{~Hz}, \operatorname{ArC}), 132.43$ (d, J $10.0 \mathrm{~Hz}, \operatorname{ArC}), 132.45$, 132.82, (d, J 1.9 Hz, ArC), 132.93, (d, J 1.8 Hz, ArC), 133.80, (d, J 9.7 Hz, ArC), 133.89 (ArC), 134.49, (d, J 6.4 Hz, ArC), 158.35, (d, J $3.7 \mathrm{~Hz}, \operatorname{ArC}$ ), 160.33 (d, J 1.8 Hz, ArC). ${ }^{31} \mathrm{P}-\mathrm{NMR}$ 
$\left(121.5 \mathrm{MHz}, \mathrm{CDCl}_{3}\right): \delta 23.35 ; \mathrm{MS}: 497.2(\mathrm{M}+\mathrm{H})^{+}$. Anal Calcd for $\mathrm{C}_{28} \mathrm{H}_{33} \mathrm{O}_{6} \mathrm{P}: \mathrm{C}, 67.73 ; \mathrm{H}, 6.70$. Found: C, 67.48; H, 6.47.

(7S,15S)-7,15-Dimethyl-22-phenyl-6,7,9,10,12,13,15,16-octahydro-22H-22 $\lambda^{5}$-dibenzo[n,q]$[1,4,7,10,13,16]$ pentaoxaphosphacyclooctadecin-22-one $[(S, S)-7]$. Triphenyl phosphanone 11 $(0.58 \mathrm{~g}, 1.9 \mathrm{mmol})$, dimethyl-substituted tetraethylene glycol ditosylate $(S, S)-\mathbf{1 2}{ }^{33}(1.00 \mathrm{~g}, 1.9$ mmol) and finely powdered anhydrous $\mathrm{K}_{2} \mathrm{CO}_{3}(2.88 \mathrm{~g}, 20.9 \mathrm{mmol})$ were mixed with vigorous stirring in dry DMF (40 mL) under Ar. The temperature of the reaction mixture was raised to 80 ${ }^{\circ} \mathrm{C}$ and kept stirring at this temperature until TLC analysis showed the total consumption of the starting materials (4 days). The solvent was removed at $40{ }^{\circ} \mathrm{C}$, the residue was suspended in water $(200 \mathrm{~mL})$ and it was extracted with $\mathrm{CH}_{2} \mathrm{Cl}_{2}(4 \times 100 \mathrm{~mL})$. The combined organic phase was shaken with $\mathrm{H}_{2} \mathrm{O}(50 \mathrm{~mL})$, dried over $\mathrm{MgSO}_{4}$, filtered and the solvent was removed. The crude product was purified by chromatography on silica gel using methanol- $\mathrm{CH}_{2} \mathrm{Cl}_{2}$ (1:30) as an eluent to give $(S, S)-7$. This product was triturated with diisopropyl ether to give a yellow solid $(0.39 \mathrm{~g}$, 41\%). mp 136-138 ${ }^{\circ} \mathrm{C}$ (from methanol). $R_{\mathrm{f}}$ : 0.34 (silica gel TLC, ethyl acetate-hexane 1:1); $[\alpha]_{\mathrm{D}}{ }^{25}+56.1$ (c 0.97, methanol); IR (neat) $v_{\max } 1161 \mathrm{~cm}^{-1} v \mathrm{P}=\mathrm{O} ;{ }^{1} \mathrm{H}-\mathrm{NMR}\left(300 \mathrm{MHz}, \mathrm{CDCl}_{3}\right) \delta$ 0.98-1.04 (m, 6H, $\left.\mathrm{CH}_{3}\right), 2.79-2.81\left(\mathrm{~m}, 1 \mathrm{H}, \mathrm{OCH}, \mathrm{OCH}_{2}\right), 3.09-3.16\left(\mathrm{~m}, 2 \mathrm{H}, \mathrm{OCH}, \mathrm{OCH}_{2}\right), 3.21$ $3.24\left(\mathrm{~m}, 1 \mathrm{H}, \mathrm{OCH}, \mathrm{OCH}_{2}\right), 3.33-3.35\left(\mathrm{~m}, 1 \mathrm{H}, \mathrm{OCH}, \mathrm{OCH}_{2}\right), 3.40-3.51\left(\mathrm{~m}, 4 \mathrm{H}, \mathrm{OCH}, \mathrm{OCH}_{2}\right)$, 3.57-3.59 (m, 1H, OCH, $\left.\mathrm{OCH}_{2}\right), 3.68-3.71\left(\mathrm{~m}, 1 \mathrm{H}, \mathrm{OCH}, \mathrm{OCH}_{2}\right), 3.78-3.81(\mathrm{~m}, 1 \mathrm{H}, \mathrm{OCH}$, $\left.\mathrm{OCH}_{2}\right), 3.93-3.96\left(\mathrm{~m}, 1 \mathrm{H}, \mathrm{OCH}, \mathrm{OCH}_{2}\right), 4.12-4.15\left(\mathrm{~m}, 1 \mathrm{H}, \mathrm{OCH}, \mathrm{OCH}_{2}\right), 6.94-7.06(\mathrm{~m}, 4 \mathrm{H}$, ArH), 7.42-7.47 (m, 6H, ArH), 7.75-7.79 (m, 1H, ArH), 7.98-8.02 (m, 2H, ArH). ${ }^{13} \mathrm{C}-\mathrm{NMR}(75$ $\left.\mathrm{MHz}, \mathrm{CDCl}_{3}\right) \delta 17.13,17.43\left(\mathrm{CH}_{3}\right)$, 69.68, 70.00, 71.32, 71.51, 72.05, 73.06, 75.18, 75.60 $\left(\mathrm{OCH}, \mathrm{OCH}_{2}\right), 112.54$ (d, J 6.75 Hz, ArC), 112.97 (d, J 6.4 Hz, ArC), 120.67 (d, J 4.7 Hz, ArC), 120.68 (d, J 17.4 Hz, ArC), 122.50 (d, J 198.1 Hz, ArC), 122.51 (d, J 16.4 Hz, ArC), 128.15 (d, $J 12.75 \mathrm{~Hz}, \mathrm{ArC}), 131.21$ (ArC), 132.21 (d, $J 10.3 \mathrm{~Hz}, \operatorname{ArC}), 133.11,133.32,134.07$, 134.17,134.64 (ArC), 160.65 (d, J 42.2 Hz, ArC). ${ }^{31} \mathrm{P}-\mathrm{NMR}\left(121.5 \mathrm{MHz}, \mathrm{CDCl}_{3}\right.$ ): $\delta$ 24.01; MS: $497.2(\mathrm{M}+\mathrm{H})^{+}$. Anal Calcd for $\mathrm{C}_{28} \mathrm{H}_{33} \mathrm{O}_{6} \mathrm{P}: \mathrm{C}, 67.73$; H, 6.70. Found: C, 67.61; H, 6.77.

\section{(7S,15S)-7,15-Dioctyl-22-phenyl-6,7,9,10,12,13,15,16,18,19-decahydro-25H-25 $\lambda^{5}$-dibenzo-} $[q, t][1,4,7,10,13,16,19]$ hexaaoxaphosphacyclooctahenicosin-22-one $\quad[(S, S)-8]$. Macrocycle $(S, S)-\mathbf{8}$ was prepared from the reported ditosylate $(S, S)-13^{33}(0.55 \mathrm{~g}, 0.76 \mathrm{mmol})$ and $\mathbf{1 1}(0.24 \mathrm{~g}$, $0.76 \mathrm{mmol})$ in the same way as described above for $(S, S)-7$. The reaction was completed in 7 days. The crude product was purified by PLC on aluminium oxide using acetone-hexane (1:2) as an eluent to give $(S, S)-8(0.241 \mathrm{~g}, 43 \%)$ as a colorless oil. $R_{\mathrm{f}}: 0.85$ (aluminium oxide TLC, acetone-hexane 1:2); $[\alpha]_{\mathrm{D}}{ }^{34}+16.18\left(\mathrm{c} 1.36, \mathrm{CH}_{2} \mathrm{Cl}_{2}\right)$; IR (neat) $v_{\max } 1163,1188 \mathrm{~cm}^{-1} v \mathrm{P}=\mathrm{O} ;{ }^{1} \mathrm{H}-$ NMR $\left(300 \mathrm{MHz}, \mathrm{CDCl}_{3}\right) \delta 0.90\left(\mathrm{t}, J 6.3 \mathrm{~Hz}, 6 \mathrm{H}, \mathrm{CH}_{3}\right), 1.09-1.27\left(\mathrm{~m}, 28 \mathrm{H}, \mathrm{CH}_{2}\right), 2.52-6.13(\mathrm{~m}$, $\left.1 \mathrm{H}, \mathrm{OCH}, \mathrm{OCH}_{2}\right), 2.84-2.92\left(\mathrm{~m}, 1 \mathrm{H}, \mathrm{OCH}, \mathrm{OCH}_{2}\right), 3.13-3.25\left(\mathrm{~m}, 2 \mathrm{H}, \mathrm{OCH}, \mathrm{OCH}_{2}\right), 3.36-3.67$ $\left(\mathrm{m}, 8 \mathrm{H}, \mathrm{OCH}, \mathrm{OCH}_{2}\right), 3.77-3.84\left(\mathrm{~m}, 2 \mathrm{H}, \mathrm{OCH}, \mathrm{OCH}_{2}\right), 3.89-4.00\left(\mathrm{~m}, 2 \mathrm{H}, \mathrm{OCH}, \mathrm{OCH}_{2}\right), 6.91-$ $7.03(\mathrm{~m}, 3 \mathrm{H}, \mathrm{ArH}), 7.03-7.11(\mathrm{~m}, 1 \mathrm{H}, \mathrm{ArH}), 7.21-7.26(\mathrm{~m}, 1 \mathrm{H}, \mathrm{ArH}), 7.42-7.54(\mathrm{~m}, 5 \mathrm{H}, \mathrm{ArH})$, 7.91-8.02 (m, 3H, ArH); ${ }^{13} \mathrm{C}-\mathrm{NMR}\left(75 \mathrm{MHz}, \mathrm{CDCl}_{3}\right) \delta 14.15,22.71,25.11,25.47,29.33,29.55$, 29.63, 29.73, 29.75, 31.90, 31.91, 32.42, $32.97\left(\mathrm{CH}_{2}, \mathrm{CH}_{3}\right), 70.19,70.58,70.61,70.83,70.99$, 71.06, 71.23, 72.28, 78.46, $78.68\left(\mathrm{OCH}, \mathrm{OCH}_{2}\right), 112.46$ (d, J 6.7 Hz, ArC), 112.76 (d, J 5.8 Hz, 
ArC), 120.69 (d, J $12.7 \mathrm{~Hz}, \quad \operatorname{ArC}), 121.17$ (d, J $12.3 \mathrm{~Hz}, \quad \operatorname{ArC}), 122.82$ (d, $J 23.4 \mathrm{~Hz}, \operatorname{ArC}$ ), 123.94 (d, J 68.2 Hz, ArC), 128.15 (d, J 12.3 Hz, ArC), 131.30, 131.33 (ArC), 131.93 (d, $J 10.0 \mathrm{~Hz}, \mathrm{ArC}$ ), 133.08, 133.12, 133.14 (ArC), 133.64 (d, $J 9.4 \mathrm{~Hz}, \operatorname{ArC}$ ), 134.06 (ArC), 134.07 (d, J 6.6 Hz, ArC), 159.68 (d, J 2.4 Hz, ArC), 160.99 (d, J 1.9 Hz, ArC). ${ }^{31} \mathrm{P}-\mathrm{NMR}$ $\left(121.5 \mathrm{MHz}, \mathrm{CDCl}_{3}\right): \delta 22.75 ; \mathrm{MS}: 737.5(\mathrm{M}+\mathrm{H})^{+}$. Anal Calcd for $\mathrm{C}_{44} \mathrm{H}_{65} \mathrm{O}_{7} \mathrm{P}: \mathrm{C}, 71.71 ; \mathrm{H}, 8.89$. Found: C, 71.96; H, 9.02.

General procedure for the reduction of phosphanones to phosphanes. Triphenylphosphanone (17) $(0.1 \mathrm{~g}, 0.36 \mathrm{mmol})$, and triethoxysilane $(2.00 \mathrm{~mL}, 10.8 \mathrm{mmol})$ were mixed with vigorous stirring in a sealed tube under Ar. The temperature of the reaction mixture was raised to $150{ }^{\circ} \mathrm{C}$ and kept stirring at this temperature for 2 days. The solvent was removed at $40{ }^{\circ} \mathrm{C}$ and the crude product was purified by PLC on silica gel using hexane as an eluent to give triphenylphosphane $18(0.082 \mathrm{~g}, 82 \%) . R_{\mathrm{f}, \mathbf{1 3}}: 0.20, R_{\mathrm{f}, \mathbf{1 4}}: 0.92$ (silica gel TLC, acetone-hexane 1:3). The product was pure according to TLC analysis, ${ }^{1} \mathrm{H}$ - and ${ }^{31} \mathrm{P}-\mathrm{NMR}$.

Triphenylphosphanone (17) $(0.1 \mathrm{~g}, 0.36 \mathrm{mmol})$, and trimethoxysilane $(2.00 \mathrm{~mL}, 15.7 \mathrm{mmol})$ were mixed with vigorous stirring in a sealed tube under Ar. The temperature of the reaction mixture was raised to $150{ }^{\circ} \mathrm{C}$ and kept stirring at this temperature for 2 days. The solvent was removed at $40{ }^{\circ} \mathrm{C}$ and the crude product was purified by PLC on silica gel using hexane as an eluent to give triphenylphosphane $18(0.086 \mathrm{~g}, 86 \%) . R_{\mathrm{f}, \mathbf{1 3}}: 0.20, R_{\mathrm{f}, \mathbf{1 4}}: 0.92$ (silica gel TLC, acetone-hexane 1:3). The product was pure according to TLC analysis, ${ }^{1} \mathrm{H}$ - and ${ }^{31} \mathrm{P}-\mathrm{NMR}$.

\section{2-Phenyl-6,7,9,10,12,13,15,16-octahydro-22H-dibenzo[n,q][1,4,7,10,13,16]pentaoxa-}

phosphacyclooctadecin (1). Macrocycle 1 was prepared from phosphanone 5 (0.16 g, 0.35 mmol) and trimethoxysilane $(2 \mathrm{~mL}, 15.7 \mathrm{mmol})$ in the same way as described above for $\mathbf{1 8}$. The reaction was completed in 4 days. The solvent was removed at $30{ }^{\circ} \mathrm{C}$ and the crude product was purified by PLC on silica gel using methanol- $\mathrm{CH}_{2} \mathrm{Cl}_{2}$ (1:40) as an eluent to give $\mathbf{1}(0.119 \mathrm{~g}, 76 \%)$ as a white solid. mp112-114 ${ }^{\circ} \mathrm{C}$ (from methanol). $R_{\mathrm{f}}$ : 0.55 (silica gel TLC, methanol- $\mathrm{CH}_{2} \mathrm{Cl}_{2}$ 1:30). IR (neat) no peak at $1166 \pm 30 \mathrm{~cm}^{-1}$ for $v \mathrm{P}=\mathrm{O} .{ }^{1} \mathrm{H}-\mathrm{NMR}\left(300 \mathrm{MHz}, \mathrm{CDCl}_{3}\right) \delta 3.66-3.85$ $\left(\mathrm{m}, 12 \mathrm{H}, \mathrm{OCH}_{2}\right), 4.04-4.18\left(\mathrm{~m}, 4 \mathrm{H}, \mathrm{OCH}_{2}\right), 6.69-6.72(\mathrm{~m}, 2 \mathrm{H}, \mathrm{ArH}), 6.83-6.91(\mathrm{~m}, 4 \mathrm{H}, \mathrm{ArH})$, 7.28-7.34 (m, 7H, ArH). ${ }^{13} \mathrm{C}-\mathrm{NMR}\left(75 \mathrm{MHz}, \mathrm{CDCl}_{3}\right) \delta 68.89,69.73,70.73,70.76,71.88,71.92$ $\left(\mathrm{OCH}_{2}\right), 111.01$ (d, J 1.1 Hz, ArC), 111.15, 121.03, 125.94 (ArC), 126.03 (d, J14.2 Hz, ArC), 128.30 (d, J 7.2 Hz, ArC), 128.50, 129.89, 133.69 (ArC), 134.34 (d, J 21.5 Hz, ArC), 136.75 (d, $J 12.9 \mathrm{~Hz}, \mathrm{ArC}$ ), 160.59 (d, $J 16.9 \mathrm{~Hz}, \mathrm{ArC}) .{ }^{31} \mathrm{P}-\mathrm{NMR}\left(121.5 \mathrm{MHz}, \mathrm{CDCl}_{3}\right.$ ): $\delta$-28.33; Anal Calcd for $\mathrm{C}_{26} \mathrm{H}_{29} \mathrm{O}_{5} \mathrm{P}: \mathrm{C}, 69.01$; H, 6.46. Found: C, 68.93. H, 6.14.

$(6 R, 16 R)-6,16-D i m e t h y l-22-p h e n y l-6,7,9,10,12,13,15,16-0 c t a h y d r o-22 H$-dibenzo[n,q]$[1,4,7,10,13,16]$ pentaoxaphosphacyclooctadecin $[(\boldsymbol{R}, \boldsymbol{R})-2]$. Macrocycle $(R, R)-2$ was prepared from phosphanone $(R, R)-6(0.11 \mathrm{~g}, 0.23 \mathrm{mmol})$ and trimethoxysilane $(2 \mathrm{~mL}, 15.7 \mathrm{mmol})$ in the same way as described above for $\mathbf{1 8}$. The reaction was completed in 4 days. The solvent was removed at $30{ }^{\circ} \mathrm{C}$ and the crude product was purified by PLC on silica gel using methanol$\mathrm{CH}_{2} \mathrm{Cl}_{2}(1: 40)$ as an eluent to give $(R, R)-2(0.0973 \mathrm{~g}, 71 \%)$ as a colorless oil. $R_{\mathrm{f}}: 0.62$ (silica gel TLC, methanol- $\mathrm{CH}_{2} \mathrm{Cl}_{2}$ 1:30). $[\alpha]_{\mathrm{D}}{ }^{29}-194.2$ (c 0.97, $\mathrm{CH}_{2} \mathrm{Cl}_{2}$ ); IR (neat) no peaks between 1130- 
$1234 \mathrm{~cm}^{-1}$ for $v \mathrm{P}=\mathrm{O} .{ }^{1} \mathrm{H}-\mathrm{NMR}\left(300 \mathrm{MHz}, \mathrm{CDCl}_{3}\right) \delta 0.91\left(\mathrm{~d}, J=6.2 \mathrm{~Hz}, 3 \mathrm{H}, \mathrm{CH}_{3}\right), 1.13(\mathrm{~d}, J=$ $\left.6.2 \mathrm{~Hz}, 3 \mathrm{H}, \mathrm{CH}_{3}\right), 3.44-3.85\left(\mathrm{~m}, 12 \mathrm{H}, \mathrm{OCH}, \mathrm{OCH}_{2}\right), 4.53-4.66\left(\mathrm{~m}, 2 \mathrm{H}, \mathrm{OCH}, \mathrm{OCH}_{2}\right), 6.72-6.94$ (m, 6H, ArH), 7.26-7.40 (m, 7H, ArH). ${ }^{13} \mathrm{C}-\mathrm{NMR}\left(75 \mathrm{MHz}, \mathrm{CDCl}_{3}\right) \delta 15.54,16.16\left(\mathrm{CH}_{3}\right), 70.50$, 70.52, 70.73, 70.75, 71.07, 71.10, 72.06, 72.10, 73.62, 73.63, 73.79, 73.80, 74.25, 74.93 (OCH, $\mathrm{OCH}_{2}$ ), 111.79 (d, J 1.7 Hz, ArC), 112.17 (d, J 1.2 Hz, ArC), 120.24 (ArC), 120.98 (d, J 1.2 Hz, ArC), 125.87 (d, J 10.9 Hz, ArC), 126.80 (d, J 12.7 Hz, ArC), 128.20 (d, J 7.5 Hz, ArC), 128.51 (ArC), 129.68 (d, J 6.0 Hz, ArC), 133.42, 134.47, 134.49, 134.53 (ArC), 134.65 (d, J $21.4 \mathrm{~Hz}$, ArC), 136.46 (d, $J 10.5 \mathrm{~Hz}, \operatorname{ArC}), 159.25$ (d, $J 13.1 \mathrm{~Hz}, \operatorname{ArC}), 159.45$ (d, J $15.5 \mathrm{~Hz}, \operatorname{ArC}) .{ }^{31} \mathrm{P}-$ NMR (121.5 MHz, $\mathrm{CDCl}_{3}$ ): $\delta$-24.03. Anal Calcd for $\mathrm{C}_{28} \mathrm{H}_{33} \mathrm{O}_{5} \mathrm{P}: \mathrm{C}, 69.98, \mathrm{H}, 6.92$. Found: C, $69.88, \mathrm{H}, 6.96$.

\section{(7S,15S)-7,15-Dioctyl-22-phenyl-6,7,9,10,12,13,15,16,18,19-decahydro-25H-dibenzo[q,t]-} $[1,4,7,10,13,16,19]$ hexaaoxaphosphacyclooctahenicosin $[(S, S)-3]$. Macrocycle $(S, S)-3$ was prepared from phosphanone $(S, S)-8(0.10 \mathrm{~g}, 0.15 \mathrm{mmol})$ and trimethoxysilane $(2 \mathrm{~mL}, 15.7$ $\mathrm{mmol}$ ) in the same way as described above for 18. The reaction was completed in 4 days. The solvent was removed at $30{ }^{\circ} \mathrm{C}$ and the crude product was purified by PLC on silica gel using methanol- $\mathrm{CH}_{2} \mathrm{Cl}_{2}(1: 60)$ as an eluent to give $(S, S)-3(0.0779 \mathrm{~g}, 78 \%)$ as a colorless oil. $R_{\mathrm{f}}: 0.88$ (silica gel TLC, methanol- $\mathrm{CH}_{2} \mathrm{Cl}_{2}$ 1:30). $[\alpha]_{\mathrm{D}}{ }^{29}+73.2$ (c $1.11, \mathrm{CH}_{2} \mathrm{Cl}_{2}$ ); IR (neat) no peaks between 1131-1239 $\mathrm{cm}^{-1}$ for $v \mathrm{P}=\mathrm{O} .{ }^{1} \mathrm{H}-\mathrm{NMR}\left(300 \mathrm{MHz}, \mathrm{CDCl}_{3}\right) \delta 0.91\left(\mathrm{t}, J 6.6 \mathrm{~Hz}, 6 \mathrm{H}, \mathrm{CH}_{3}\right)$, 1.20-1.45 (m, 28H, CH $), 3.22-3.29\left(\mathrm{~m}, 1 \mathrm{H}, \mathrm{OCH}, \mathrm{OCH}_{2}\right), 3.45-3.52\left(\mathrm{~m}, 2 \mathrm{H}, \mathrm{OCH}, \mathrm{OCH}_{2}\right)$, 3.60-3.70 (m, 10H, OCH, $\left.\mathrm{OCH}_{2}\right), 3.78-4.05\left(\mathrm{~m}, 5 \mathrm{H}, \mathrm{OCH}, \mathrm{OCH}_{2}\right), 6.60-6.63(\mathrm{~m}, 1 \mathrm{H}, \operatorname{ArH})$, 6.77-6.87 (m, 5H, ArH), 7.31-7.34 (m, 7H, ArH); ${ }^{13} \mathrm{C}-\mathrm{NMR}\left(75 \mathrm{MHz}, \mathrm{CDCl}_{3}\right) \delta 14.17\left(\mathrm{CH}_{3}\right)$, 22.73, 25.22, 25.65, 29.34, 29.60, 29.63, 29.67, 31.86, 31.93, $32.23\left(\mathrm{CH}_{2}\right), 70.32,70.37,70.50$, 70.56, 71.30, 71.40, 71.88, 71.92, 78.67, $78.71\left(\mathrm{OCH}, \mathrm{OCH}_{2}\right), 110.58(\mathrm{~d}, J 13.1 \mathrm{~Hz}, \operatorname{ArC})$, 110.56, 115.55 (ArC), 120.89 (d, J 34.9 Hz, ArC), 125.24 (d, J 12.9 Hz, ArC), 125.91 (d, J 14.7 $\mathrm{Hz}, \mathrm{ArC}$ ), 128.30 (d, J 7.4 Hz, ArC), 128.64 (ArC), 129.85 (d, J 5.1 Hz, ArC), 133.22, 133.72, 133.73 (ArC), 134.51 (d, J 21.6 Hz, ArC), 136.43 (d, J 11.7 Hz, ArC), 160.37 (d, J 4.5 Hz, ArC), 160.58 (d, J 3.3 Hz, ArC). ${ }^{31} \mathrm{P}-\mathrm{NMR}\left(121.5 \mathrm{MHz}, \mathrm{CDCl}_{3}\right)$ : $\delta$-24.99; Anal Calcd for $\mathrm{C}_{44} \mathrm{H}_{65} \mathrm{O}_{6} \mathrm{P}: \mathrm{C}, 73.30 ; \mathrm{H}, 9.09$. Found: C, 73.12; H, 8.97.

\section{(7S,15S)-7,15-Dimethyl-22-phenyl-6,7,9,10,12,13,15,16-octahydro-22H-dibenzo[n,q]-}

$[1,4,7,10,13,16]$ pentaoxaphosphacyclooctadecin $[(S, S)-4]$. Macrocycle $(S, S)-4$ was prepared from phosphanone $(S, S)-7(0.10 \mathrm{~g}, 0.20 \mathrm{mmol})$ and triethoxysilane $(2 \mathrm{~mL}, 10.8 \mathrm{mmol})$ in the same way as described above for 18. The reaction was completed in 4 days. The crude product was purified by PLC on silica gel using methanol- $\mathrm{CH}_{2} \mathrm{Cl}_{2}(1: 40)$ as an eluent to give $(S, S)-4$ $(0.054 \mathrm{~g}, 56 \%)$ as a yellow oil. $R_{\mathrm{f}}: 0.62$ (silica gel TLC, ethyl acetate-hexane $\left.1: 1\right) ;[\alpha]_{\mathrm{D}}{ }^{25}+111.18$ (c $0.85, \mathrm{CH}_{2} \mathrm{Cl}_{2}$ ); IR (neat) no significant peaks between $1130-1236 \mathrm{~cm}^{-1}$ for $v \mathrm{P}=\mathrm{O}$. ${ }^{1} \mathrm{H}-\mathrm{NMR}$ $\left(300 \mathrm{MHz}, \mathrm{CDCl}_{3}\right) \delta 1.06\left(\mathrm{~d}, J 6.4 \mathrm{~Hz}, 3 \mathrm{H}, \mathrm{CH}_{3}\right), 1.11\left(\mathrm{~d}, J 6.4 \mathrm{~Hz}, 3 \mathrm{H}, \mathrm{CH}_{3}\right), 3.49-4.04(\mathrm{~m}, 3 \mathrm{H}$, OCH, $\left.\mathrm{OCH}_{2}\right), 4.14-4.19\left(\mathrm{~m}, 1 \mathrm{H}, \mathrm{OCH}, \mathrm{OCH}_{2}\right), 6.61-6.65(\mathrm{~m}, 1 \mathrm{H}, \mathrm{ArH}), 6.77-6.88(\mathrm{~m}, 5 \mathrm{H}, \mathrm{ArH})$, 7.31-7.35 (m, 7H, ArH). ${ }^{13} \mathrm{C}-\mathrm{NMR}\left(75 \mathrm{MHz}, \mathrm{CDCl}_{3}\right) \delta 17.08,17.72\left(\mathrm{CH}_{3}\right), 70.26,70.27,70.30$, 70.33, 73.04, 74.02, 74.64, $74.88\left(\mathrm{OCH}, \mathrm{OCH}_{2}\right), 110.91$ (d, J 1.2 Hz, ArC), 111.06 (d, J 1.7 Hz, ArC), 121.09 (d, J 33.0 Hz, ArC), 125.77 (d, J 13.2 Hz, ArC), 126.36 (d, J 14.7 Hz, ArC), 
128.43 (d, J 7.4 Hz, ArC), 128.70 (ArC), 130.00 (d, J 4.1 Hz, ArC), 133.25, 134.04, 134.44, 134.72 (ArC), 136.83 (d, J 12.5 Hz, ArC), 160.59 (d, J 1.4 Hz, ArC), 160.81 (d, J 2.8 Hz, ArC).

${ }^{31} \mathrm{P}-\mathrm{NMR}\left(121.5 \mathrm{MHz}, \mathrm{CDCl}_{3}\right): \delta$-26.99. Anal Calcd for $\mathrm{C}_{28} \mathrm{H}_{33} \mathrm{O}{ }_{5} \mathrm{P}: \mathrm{C}, 69.98, \mathrm{H}, 6.92$. Found: C, 69.79, H, 6.81.

\section{Acknowledgements}

Financial supports of the Hungarian Scientific Research Fund (OTKA K81127) and the New Széchenyi Development Plan (TÁMOP-4.2.1/B-09/1/KMR-2010-0002) are gratefully acknowledged. The authors thank Dr. József Nagy and Dr. György T. Balogh for their helpful discussions.

\section{References}

1. Reppe, W.; Schweckendiek, W. J. Justus Liebigs Annalen der Chemie 1948, 560, 104. http://dx.doi.org/10.1002/jlac.19485600104

2. Young, J. F.; Osborn, J. A.; Jardine, F. H.; Wilkinson, G. Chem. Comm. (London) 1965, 131.

3. Dang, T. P.; Kagan, H. B. J. Chem. Soc. D: Chem. Comm. 1971, 481.

4. Ohta, T.; Takaya, H.; Kitamura, M.; Nagai, K.; Noyori, R. J. Org. Chem. 1987, 52, 3174. http://dx.doi.org/10.1021/jo00390a043

5. Noyori, R.; Ohkuma, T.; Kitamura, M.; Takaya, H.; Sayo, N.; Kumobayashi, H.; Akutagawa, S. J. Am. Chem. Soc. 1987, 109, 5856.

http://dx.doi.org/10.1021/ja00253a051

6. Kitamura, M.; Ohkuma, T.; Inoue, S.; Sayo, N.; Kumobayashi, H.; Akutagawa, S.; Ohta, T.; Takaya, H.; Noyori, R. J. Am. Chem. Soc. 1988, 110, 629. http://dx.doi.org/10.1021/ja00210a070

7. Boezio, A. A.; Pytkowicz, J.; Cote, A.; Charette, A. B. J. Am. Chem. Soc. 2003, 125, 14260. http://dx.doi.org/10.1021/ja038291+

8. Brauer, David J.; Kottsieper, Konstantin W.; Roßenbach, S.; Stelzer, O. Eur. J. Inorg. Chem. 2003, 1748.

http://dx.doi.org/10.1002/ejic.200200388

9. Brunner, H.; Zettlmeier, W. Handbook of Enantioselective Catalysis with Transition Metal Compounds; VCH: Weinheim 1993.

10. Botteghi, C.; Marchetti, M.; Paganelli, S. in Transition Metals for Organic Synthesis; Beller M.; Bolm C. Eds.; Wiley-VCH: Weinheim 1998, pp 25-48.

11. Kollar, L.; Keglevich, G. Chem. Rev. 2010, 110, 4257.

http://dx.doi.org/10.1021/cr900364c

12. DelDonno, T. A.; Rosen, W. J. Am. Chem. Soc. 1977, 99, 8051. 
http://dx.doi.org/10.1021/ja00466a048

13. Ciampolini, M.; Dapporto, P.; Nardi, N.; Zanobini, F. J. Chem. Soc.: Chem. Comm. 1980, 177.

14. Van Zon, A.; Torny, G. J.; Frijns, J. H. G. Recl. Trav. Chim. Pays-Bas 1983, 102, 326. http://dx.doi.org/10.1002/recl.19831020609

15. Huszthy, P.; Farkas, V.; Tóth, T.; Székely, G.; Hollósi, M. Tetrahedron 2008, 64, 10107. http://dx.doi.org/10.1016/j.tet.2008.07.111

16. Maier, L. Organic Phosphorus Compounds; Wiley: New York, 1972.

17. Engel, R. in Handbook of Organophosphorus Chemistry; Engel, R. Ed. Marcel Dekker, New York. 1992, p 193.

18. Fritzsche, H.; Hasserodt, U.; Korte, F. Chemische Berichte 1964, 97, 1988. http://dx.doi.org/10.1002/cber.19640970729

19. Fritzsche, H.; Hasserodt, U.; Korte, F. Chemische Berichte 1965, 98, 171. http://dx.doi.org/10.1002/cber.19650980122

20. Myers, D. K.; Quin, L. D. J. Org. Chem. 1971, 36, 1285. http://dx.doi.org/10.1021/jo00808a025

21. Quin, L. D.; Caster, K. C.; Kisalus, J. C.; Mesch, K. A. J. Am. Chem. Soc. 1984, 106, 7021. http://dx.doi.org/10.1021/jo00808a025

22. Quin, L. D.; Szewczyk, J. Phosphorous and Sulfur and the Related Elements 1984, $21,161$. http://dx.doi.org/10.1080/03086648408077652

23. Coumbe, T.; Lawrence, N. J.; Muhammad, F. Tetrahedron Letters 1994, 35, 625. http://dx.doi.org/10.1016/S0040-4039(00)75855-2

24. Montel, S.; Jia, T.; Walsh, P. J. Org. Lett. 2014, 16, 130. http://dx.doi.org/10.1021/ol403124g

25. Trudeau, S.; Morken, J. P. Tetrahedron 2006, 62, 11470. http://dx.doi.org/10.1016/j.tet.2006.05.043

26. Snieckus, V.; Demchuk, O.; Yoruk, B.; Blackburn, T. Synlett 2006, 2908.

27. Vandyck, K.; Matthys, B.; Willen, M.; Robeyns, K.; Van Meervelt, L.; Van der Eycken, J. Org. Lett. 2006, 8, 363.

http://dx.doi.org/10.1021/o10522788

28. Odinets, I. L.; Vinogradova, N. M.; Matveeva, E. V.; Golovanov, D. D.; Lyssenko, K. A.; Keglevich, G.; Kollár, L.; Roëschenthaler, G.-V.; Mastryukova, T. A. J. Organometall. Chem. 2005, 690, 2559. http://dx.doi.org/10.1016/j.jorganchem.2004.09.030

29. Ngo, H. L.; Lin, W. J. Org. Chem. 2005, 70, 1177. http://dx.doi.org/10.1021/jo048333s

30. Hatano, M.; Horibe, T.; Ishihara, K. Angew. Chem. Int. Ed. Engl. 2013, 52, 4549. http://dx.doi.org/10.1002/anie.201300938

31. Keglevich, G.; Kovács, A.; Tőke, L.; Újszászy, K.; Argay, G.; Czugler, M.; Kálmán, A. Heteroatom Chem. 1993, 4, 329. 
http://dx.doi.org/10.1002/hc.520040405

32. Tanke, R. S.; Holt, E. M.; Crabtree, R. H. Inorg. Chem. 1991, 30, 1714.

http://dx.doi.org/10.1021/ic00008a009

33. Székely, G.; Csordás, B.; Farkas, V.; Kupai, J.; Pogány, P.; Sánta, Z.; Szakács, Z.; Tóth, T.; Hollósi, M.; Nyitrai, J.; Huszthy, P. Eur. J. Org. Chem. 2012, 3396.

http://dx.doi.org/10.1002/ejoc.201101769

34. Dhawan, B.; Redmore, D. J. Org. Chem. 1986, 51, 179.

http://dx.doi.org/10.1021/jo00352a010

35. Riddick, J. A.; Bunger, W. B.; Sakano, T. K. Organic Solvents: Physical Properties and Methods of Purification; Wiley-Interscience: New York, 1986. 